\title{
Análisis de la investigación sobre Radio en España: una aproximación a través del Análisis Bibliométrico y de Redes Sociales de las tesis doctorales defendidas en España entre 1976-2008
}

\author{
Rafael REPISO CABALLERO \\ ESCO (Escuela Superior de Comunicación de Granada) \\ Centro adscrito a la Universidad de Gales) \\ rrepiso@escogranada.com \\ Daniel TORRES SALINAS \\ Universidad de Navarra \\ torressalinas@gmail.com \\ Emilio Delgado LóPEZ-CóZAR \\ Universidad de Granada \\ edelgado@ugr.es
}

Recibido: $22 / 02 / 2011$

Aceptado: 17/10/2011

\begin{abstract}
Resumen
Estudio descriptivo longitudinal retrospectivo de las tesis doctorales sobre radio, defendidas en España entre 1976 y 2008. Los datos han sido extraídos principalmente de Teseo. A fin de fijar y representar la evolución, participación de universidades, perfil de los actores, genealogías académicas, focos y grupos invisibles de trabajo e investigación se han estudiado las siguientes variables: Frecuencia de términos de los títulos, evolución del número de tesis leídas por periodos, universidad de lectura, dirección de tesis así como la presidencia del mismo y genealogías director-doctorando. El análisis de redes vislumbra y visualiza las interacciones entre académicos, el nivel de independencia de los diferentes actores de la red, la coparticipación entre actores en tribunales, el grado de intermediación de los diferentes académicos.
\end{abstract}

Palabras clave: Análisis de redes sociales, comunicación audiovisual, radio, investigación, bibliometría.

\section{Transformations in the models of journalists formation in Spain}

\begin{abstract}
This is a longitudinal, retrospective and descriptive study about doctoral theses on radio which have been defended in Spain over the period 1976-2008. The TESEO database has been used to search theses and the composition of examination boards for theses on radio, even when the data had been contrasted with previous works. A number of variables have been studied to show and represent the evolution, the partnership of universities, the participants' profiles, the academic genealogies, invisibles working parties and research teams. These are the variables studied: frequency of the terms in the titles, development of the number of thesis by year, university of thesis defense, thesis advisor, participation in examination committees and advisor-candidate genealogies. The network analysis shows the interactions among professors, level of independence, cooperation among the members in the examination boards and intermediation. The membership within the theses examination boards in Spain can be observed on the graph. It shows a mode-2 social network of advisors and universities.
\end{abstract}

Keywords: Radio, Social Network Analysis, Thesis, Dissertations, Bibliometry, Audiovisual Communication, Research.

\section{Referencia normalizada}

REPISO CABALLERO, Rafael; TORRES SALINAS, Daniel; y DELGADO LÓPEZ-CÓZAR, Emilio (2011): “Análisis de la investigación sobre Radio en España: Una aproximación a través del Análisis Bi- 
bliométrico y de Redes Sociales de las tesis doctorales defendidas en España entre 1976-2008”. Estudios sobre el mensaje periodístico. Vol. 17, núm. 2, págs.: 417-429. Madrid, Servicio de Publicaciones de la Universidad Complutense.

Sumario: 1. Introducción. 2. Metodología. 3. Resultados. 3.1. Análisis bibliométrico: producción de tesis. 3.2. Análisis bibliométrico: universidades de lectura. 3.3. Análisis bibliométrico: dirección de tesis (Directores y Genealogías). 3.4. Análisis bibliométrico: presencia en tribunales y presidencia. 3.5. Análisis de Redes Sociales. 3.5.1. Medidades de centralidad: grado, cercanía e intermediación. 3.5.2. Grafos. 4. Conclusiones. 5. Referencias bibliográficas.

\section{Introducción}

No es baladí el estudio de las tesis producidas dentro un área de Conocimiento. Es profundamente interesante su análisis minucioso porque son fruto de unas dinámicas internas y externas, que salen a la luz si se les aplica el método de estudio adecuado. Actualmente la tesis doctoral se considera el primer trabajo científico que realiza el investigador. La inexperiencia del autor hace necesaria la existencia de acompañamiento en este proyecto, y más específicamente en la figura del director, que guiará al doctorando en su primer contacto con la investigación científica.

La figura del director se debe caracterizar por una serie de condiciones imprescindibles, por las que es seleccionado por el doctorando. El director debe ser un experto en la materia a investigar, tener conocimientos demostrados sobre el tema y la metodología necesaria. Así mismo debe ser capaz de comprometerse con un proyecto que puede prolongarse años.

La característica principal de la Tesis es que es un trabajo de investigación original que aporta un conocimiento científico y especializado inédito. Para evaluar este trabajo se crean sistemas de control y evaluación mediante la composición de tribunales de profesores e investigadores doctos en la materia. La selección de los miembros del tribunal, al igual que la selección del director de la tesis está cargada de significado. Ello hace que los datos que TESEO guarda sean muy valiosos para la investigación de las estructuras sociales de los sistemas de investigación nacionales en las diferentes disciplinas.

Como ejemplo se pueden citar los trabajos en las siguientes areas: Anestesiología (Figueredo et Al., 2002), Psicología (Pérez, Mestre, 1991) (Civera Molla, TortOSA GiL, 2001) (AGUdELO ET AL., 2002), Reumatología, Geografía (LÁZARO TORRES, 2002) Rehabilitación (MuÑoz Tinoco, 1998), Cuidados paliativos (CENTENo, 2001), Biblioteconomía y Documentación (Delgado López-CózAR, 2003) (Delgado LóPEzCózar, et AL., 2006) (Fuentes i Pujol; GonzÁlez Quesada, 2002) (López-Yepes, 2002), Ornitología (BARbosa, 2000), Educación Física (Ponce De León EliZONDO,1999), Matemática (Fernández CANO, ET AL.,2003, 2008) (VAllejo Ruiz ET AL., 2008), Estudios sobre la mujer (ToRRES-RAMírEZ, ToRRES-SALINAS, 2005, 2007). El reciente artículo de FuENTES I PUJOL (2010) recopila y clasifica la mayor parte de trabajos relacionadas con el área.

Actualmente no existe ningún trabajo descriptivo sobre la investigación en Radio en España, sino es formando parte de estudios más amplios sobre Comunicación, y tratándose de la Radio, de una forma superficial (AgudELo et al., 2002) (Aguilera MoyANO, 1998) (ÁlLVAREZ, 1993) (MARTínEZ Nicolás, 2006). Cabe destacar la labor de 
Daniel E. JONES cuyo interés en la medición y comprensión de la investigación en Comunicación ha legado un gran cuerpo doctrinal de artículos y libros $(1994,1997,1998$, 1999, 2000) del cual este artículo es en parte deudor.

El objetivo principal de este artículo es analizar el estado de la investigación española en Radio reflejado en las tesis doctorales que se han presentado en el periodo 1976-2008, producción por universidades, periodos, directores, genealogías, etc. El año de inicio del estudio viene dado porque Teseo recopila tesis desde 1976. Así mismo también se trata de identificar a los actores principales del sistema mediante la cuantificación de la producción y el Análisis de Redes Sociales (Social Network Analysis).

\section{Metodología}

Este trabajo es un estudio descriptivo longitudinal retrospectivo de las tesis doctorales españolas sobre Radio leídas en las universidades desde el año 1976 hasta el 2008. Para ello se ha localizado la información principalmente en TESEO. La base de datos contiene los siguientes campos; Autor, Título de la tesis, Departamento de Lectura ${ }^{1}$, Fecha de Lectura ${ }^{2}$, Director de tesis ${ }^{3}$, Universidad, Vocales y Descriptores ${ }^{4}$. Esta base de datos se distribuye en línea desde 1997 y contiene tesis doctorales leídas y aprobadas en las universidades españolas desde el año 1976. Para estudios más amplios como el de Jones, et. al.: 2000 se debe recurrir a métodos más costosos, en tiempo y dinero. Igualmente, como herramienta de validación se ha usado este estudio para completar la muestra.

La información se introduce en TESEO enviada por la universidad, a través de la secretaría del tribunal de tesis o del doctorando, en un principio remitida al Ministerio por la Comisión de Doctorado desde 1985 y posteriormente por la secretaría de la facultad donde ha tenido lugar la lectura. Actualmente es el doctorando el que debe inscribir su tesis en TESEO. Aparentemente TESEO no normaliza con profundidad sus datos, lo que se traduce en registros sin materias asignadas, inexistencia de un control de autoridades en nombres propios, departamentos, etc. Si a todo esto se le suma la "caducidad" de los nombres de los departamentos y facultades, que son renombrados periódicamente, se dificulta especialmente la labor de búsqueda, recuperación y normalización de los datos.

TESEO no es una base de datos exhaustiva, hay tesis omitidas, muchos registros están incompletos, existen registros duplicados y la incorporación y actualización de la base de datos es lenta. Es importante conocer la problemática que ofrece TESEO, pues el uso acrítico de esta fuente puede conducir a conclusiones erróneas.

La estrategia de búsqueda viene condicionada por la falta de uso en los descriptores de materia en TESEO. Así pues, se planteó una búsqueda temática en el título y en el resumen. Para ello se buscaron todas las tesis de Radio surgidas en los departa-

${ }^{1}$ Este dato no aparece en la mayoría de los registros, solamente se han recuperado el 46\%.

${ }^{2}$ En muchos casos la fecha concreta es orientativa, probablemente porque se incorporaron los registros con posterioridad. Solamente el año de lectura parece fiable.

${ }^{3}$ Hay 7 tesis sin director.

${ }^{4}$ Debido a la falta de normalización de materias en TESEO, este campo es anecdótico. 
mentos relacionados con los estudios de Comunicación Audiovisual, es decir se realizó una búsqueda por departamentos. Posteriormente, se analizaron los términos usados en los títulos de las tesis recogidas y se planteó una segunda búsqueda temática por el título. Se extrajeron los siguientes términos: Radio/Radiodifusión/Radiofónico/a/Emisoras/Emisión. La búsqueda se hizo truncando los términos y por periodos de años, ya que el término "Radio" devolvía más resultados de los que la base de datos permite consultar (esta búsqueda devolvía 4131 tesis y sólo permite visualizar 250).

Esta estrategia de búsqueda recuperó 97 tesis, algunas de las cuales debieron ser completadas por la ausencia de datos como el director o el tribunal. Es posible encontrar al director de una tesis en el registro catalográfico de la universidad en la que está depositada, en cambio es muy difícil rehacer el tribunal si este dato faltase. Como último paso se contrastaron con las tesis que Daniel E Jones y otros autores (2000) recopilaron en el libro "Análisis bibliométrico de las tesis sobre comunicación en España", y se añadieron 21 tesis que no estaban registradas en TESEO; algunas tan necesarias y representativas como las de Enrique Bustamante, Rosa Franquet y Armand Balsebre entre otros. El número de tesis final es de 118, aunque muy probablemente este número no coincida con exactitud con el de las tesis leídas sobre Radio en las universidades, principalmente por defectos de cobertura de base, si puede decirse que las tesis recuperadas son lo suficientemente representativas gracias a la exhaustividad de la búsqueda, el control de la normalización y el contraste con otros trabajos. Este conjunto de tesis muestra, sin lugar a dudas, las tendencias de producción, aunque la estructura social plasmada en las redes puede introducir algunos sesgos. Todos los datos obtenidos se exportaron a Microsoft ${ }^{\circledR}$ Exxcel 2007 para su análisis bibliométrico. Se usó Microsoft ${ }^{\circledR}$ Access 2007 para migrar los datos a Pajek 1.0.6. con el que se realizó el análisis de redes.

En el ARS (Análisis de Redes Sociales) se analizaron indicadores descriptivos básicos, entre ellos densidad y medidas de centralidad.

Densidad: Entendida como la proporción de relaciones existentes sobre el total posible. Este indicador informa sobre la cohesión de la red

Centralidad: Cuyo objetivo es estudiar los actores centrales, definido en poder, prominencia sobre el grupo y prestigio. Definir el poder en un grupo es complejo, pero en al análisis de Redes Sociales podemos afirmar que el poder es inherentemente relacional, Como el grado (Degree), la cercanía (Closseness) y la intermediación (Betweeness).

Grado (Degree): Número total de vínculos directos que tiene un nodo con otros nodos en la red.

El grado de cercanía (Closseness) mide la distancia media de proximidad de cada actor con respecto al resto de actores de la red. Un alto grado indica una facilidad de acceso al resto de los actores de la red. En definitiva, una mayor posibilidad de comunicarse. El poder de la cercanía se explica en la medida en que a un actor se le ofrecen posibilidades o se le imponen restricciones.

El grado de intermediación (Betweeness). Medido en cada actor de la red nos indica la función interconectora de conexiones geodésicas (es decir, las más inmediatas) del mismo entre el resto de actores. Los actores con el mayor grado de intermediación alcanzan mayor "poder" porque están presentes en las conexiones más importantes. 
Los actores con mayor intermediación conectan a otros, pero también tienen la capacidad de aislarlos, debido a la dependencia.

Por último se incluyen dos gráficas de redes. La primera es una gráficas de modo1(una clase única de nodos) donde el tamaño de los nodos representa el grado nodal de cada actor.que representa. Esta gráfica han sido "podadas" aquellas relaciones con valores igual a 1, igualmente también se eliminaron aquellos nodos cuyas relaciones no tenían un valor superior a 1 . Con esto se pretende reducir la complejidad, conservando lo fundamental, el núcleo de actores con mucha presencia en tribunales y que se interrelaciona con frecuencia (al menos 2 veces). La primera gráfica de red se genera con el vector de grado del subgrupo (el grupo inicial una vez que se ha eliminado aquellas relaciones con valor igual a 1). Para la representación de esta red se ha utiliza el algoritmo de Kamada-Kawai, levemente modificado con el fin de que se aprecien las leyendas de los nodos. El algoritmo de Kamada-Kawai, que trata de minimizar la energía de los nodos, hace que la distancia entre dos nodos sea lo más fiel posible a la distancia a través de nodos y arcos.

La segunda gráfica de red es de modo-2, ya que incluye dos tipos diferentes de nodos: Universidad y Directores de tesis. Muestra la relación Director/Universidad en las 6 universidades más productivas.

En la Gráfica 1 se representan los términos de los títulos respecto a su frecuencia de aparición, con la excepción de "Radio" y "Radios" que han sido reducidos de tamaño, ya que solamente la palabra Radio tenía un tamaño relativo similar al conjunto de la gráfica. Este gráfico tiene un valor meramente representativo y ha sido generado con el programa Wordle ${ }^{\circledR}$ de IBM.

Gráfico 1. Cloud-Tag. Nube de palabras de los títulos de las tesis sobre Radio.1976-2008

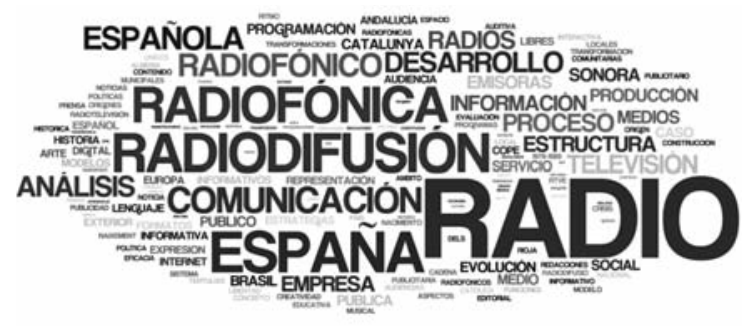

\section{Resultados}

\subsection{Análisis bibliométrico: producción de tesis}

El número total de tesis obtenidas es de 118. La primera tesis la realizó el profesor Enrique Bustamante en el año 1980 (Bustamante RAmíREZ, 1980). De 1983 a 1992 la producción de tesis se mantiene estable. El análisis de la evolución productiva evidencia una duplicación del número de tesis defendidas en el periodo 1993-1997 con respecto a 1988-1992; y 1998-2002 con respecto a 1993-1997. En el último periodo de tiempo 2003-2007 se evidencia una caída en la producción, hasta la mitad en relación al periodo anterior. El número de tesis se empieza a incrementar a mediados de los años noventa. La tendencia siempre permanece en alza, salvo en el último periodo, que aún así es el segundo periodo con mayor producción. 
Las tendencias productivas de las principales universidades protagonistas se aprecian en la tabla 1 , donde las universidades históricas en estudios de comunicación (Universidad Complutense de Madrid, Universidad Autónoma de Barcelona, Universidad de Navarra y Universidad del Pais Vasco) acumulan un $72 \%$ de las tesis totales.
Gráfico 2. No de tesis sobre radio leídas en España según quinquenios. 1982-2007

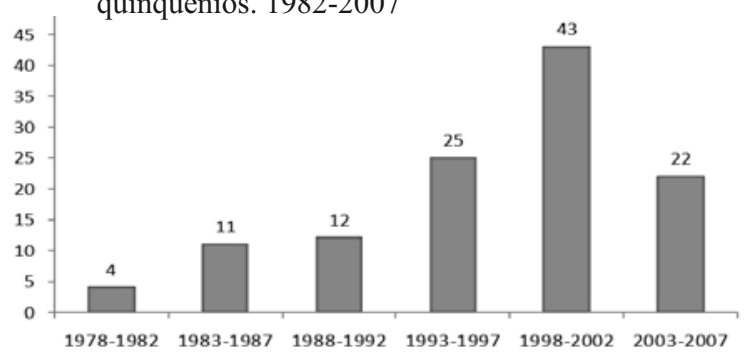
Tanto en la gráfica 2 como en la tabla 1 podemos ver cómo la tendencia alcista concuerda con mediados de los noventa, años donde se empezaron a crear nuevas facultades de Comunicación Audiovisual, así como con la potenciación de la inversión por parte del Estado en investigación, representado entre otros factores por el incremento sustancial del número de becas pre-doctorales.

Las nuevas facultades necesitan profesores especializados. Es común ante la creación de nuevos estudios la existencia de unos años de bonanza que se refleja en un aumento inusual del número de tesis. La evolución de tesis la ha encabezado la UCM en cinco de los seis periodos totales.

\subsection{Análisis bibliométrico: universidades de lectura}

Las tesis se han defendido en 24 universidades diferentes. Aunque hay una gran dispersión, algo más del $80 \%$ de las tesis las han producido solamente 6 universidades. Liderando las universidades productoras está la Universidad Complutense de Madrid, seguidas por otras, que al igual que ésta son las primeras que ofertaron estudios en Comunicación Audiovisual, e igualmente son las históricas en estudios de Comunicación. Al final del ranking nos encontramos con un grupo de 15 universidades poco productivas, la suma de las cuales representa el $13 \%$ del total.

Tabla 1. $\mathrm{N}^{\circ}$ de tesis sobre radio leídas en las Universidades Españolas por períodos.1976-2008

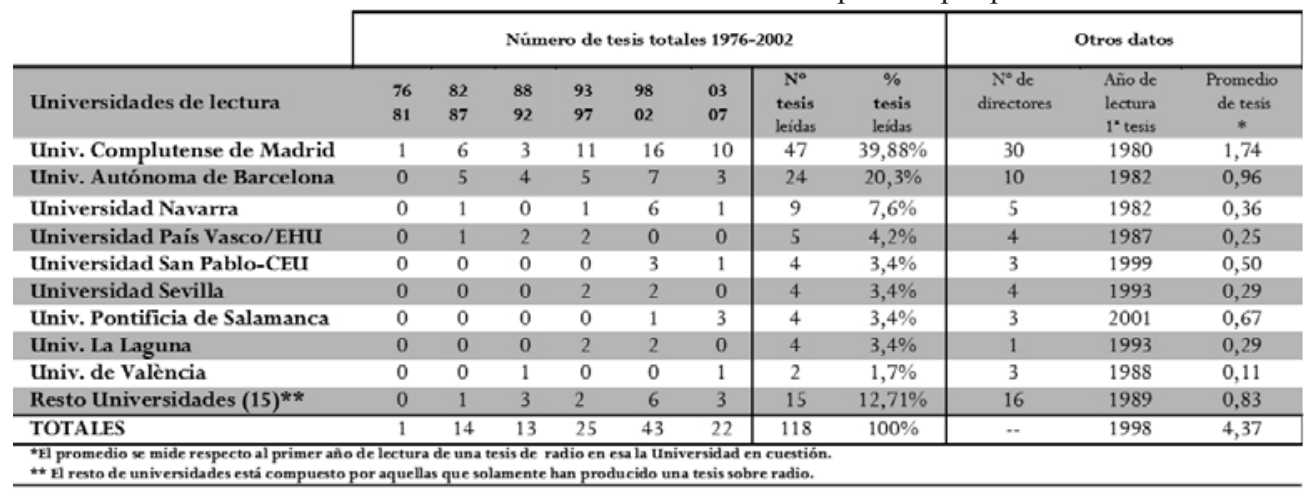

Esta baja productividad se puede deber a que son facultades en las que no hay cursos de postgrado en Comunicación Audiovisual, pero sí realizan tesis sobre radio en otras disciplinas. 
La Universidad Complutense de Madrid ha producido el $40 \%$ de las tesis, seguida por la Universidad Autónoma de Barcelona con el $20 \%$, y en tercer lugar la Universidad de Navarra con el 7\%. No es casualidad que sean las primeras universidades en impartir carreras de Comunicación.

\subsection{Análisis bibliométrico: dirección de tesis (directores y genealogías)}

Tabla 2. Directores con más de tres tesis dirigidas

\begin{tabular}{|c|c|c|c|c|c|}
\hline & & $\begin{array}{l}\mathrm{N}^{\circ} \text { Tesis } \\
\text { dirigidas }\end{array}$ & $\begin{array}{l}\% \text { Tesis } \\
\text { dirigidas } \\
\text { total* }\end{array}$ & $\begin{array}{c}\text { \% Tesis } \\
\text { Dirigidas } \\
\text { Universidad }\end{array}$ & $\begin{array}{l}\text { Año dirección } \\
\text { primer y última } \\
\text { tesis }\end{array}$ \\
\hline Ventín Pereira, José Augusto & UCM & 8 & $6,78 \%$ & $20 \%$ & $1986-2005$ \\
\hline Prado Picó, Emilio & UAB & 6 & $5,08 \%$ & $25 \%$ & 1984-1995 \\
\hline Cebrián Herreros, Mariano & UCM & 4 & $3,39 \%$ & $8,5 \%$ & $1996-2000$ \\
\hline Franquet Calvet, Rosa & UAB & 4 & $3,39 \%$ & $16,66 \%$ & 1996-2007 \\
\hline Acirón Royo, Ricardo & ULL & 4 & $3,39 \%$ & $100 \%$ & 1993-1998 \\
\hline Balsebre Torroja, Armand & UAB & 3 & $2,54 \%$ & $12,5 \%$ & 1993-2002 \\
\hline Martí Martí, Josep María & UAB & 3 & $2,54 \%$ & $12,5 \%$ & $1995-2000$ \\
\hline Merayo Pérez, Arturo & UPS & 3 & $2,54 \%$ & $75 \%$ & 2001-2005 \\
\hline Total resto directores (56) & --- & 84 & $71,19 \%$ & --- & $1980-2007$ \\
\hline TOTALES & & 118 & $100 \%$ & $-\cdots$ & $1980-2007$ \\
\hline
\end{tabular}

* El porcentaje está reducido a dos decimales.

NOTAS: UCM: Universidad Complutense de Madrid || UAB: Universidad Autónoma de Barcelona | | ULL: Universidad de la Laguna.

| U UPS: Universidad Pontificia de Salamanca. | |

En total existen 78 directores que han dirigido 111 tesis (hay 7 tesis de las que no se ha podido recuperar este datos). 7 tesis han tenido codirecciones de dos directores, ninguna de tres codirectores. El porcentaje de autores con una sola dirección es del $71.79 \%$, dos direcciones $15,38 \%$, tres direcciones $5,13 \%$, cuatro direcciones $5,13 \%$ y solamente hay dos directores con valores superiores a cuatro. Emilio Prado Picó con 6 tesis dirigidas, y José Augusto Ventín Pereira con 8 tesis dirigidas.

Se han descubierto 12 genealogías, 10 de ellas son de dos generaciones y 2 de ellas de tres generaciones. Estas últimas son:

a) La formada por Emilio Prado Picó, que es además la genealogía con mayor descendencia, con once descendientes. Seis descendientes de primera línea y cinco de segunda.

b) La genealogía de Arturo Merayo Pérez que incluye dos co-direcciones, una de ellas con Emma Rodero Antón, a la cual también le dirigió la tesis. En teoría la endogamia científica resalta los rasgos comunes.

Gráfico 3. Genealogía académica de Emilio Prado Picó y Francisco Iglesias González

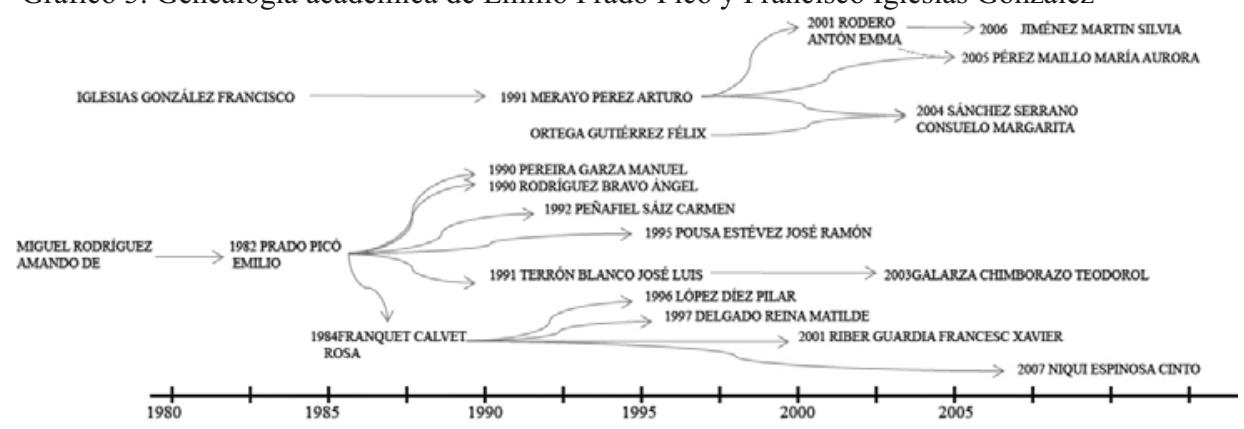




\subsection{Análisis bibliométrico: presencia en tribunales y presidencia.}

En nuestros tribunales existen 307 académicos diferentes (tenemos que recordar que este dato no ha sido recuperado en todas las tesis), de los cuales el $75 \%$ sólo ha estado en un tribunal, y un $25 \%$ ha estado en dos o más tribunales. La élite la forman los diez profesores caracterizados en la tabla 3.

El análisis de la presidencia nos muestra quiénes son aquellos académicos más solicitados: son los profesores Armand Balsebre y Mariano Cebrián quienes han ocupado mayor número de veces la presidencia. Como dato añadido, estos dos profesores nunca han co-participado en ningún tribunal del estudio.

Tabla 3. Académicos con más de 6 presencias en tribunales o 3 presidencias. Periodo 1976-

\begin{tabular}{|c|c|c|c|c|c|}
\hline Nombre & UINIV & $\begin{array}{c}\mathrm{N}^{\circ} \mathrm{de} \\
\text { presencias } \\
\text { en tribunales }\end{array}$ & $\begin{array}{c}\mathrm{N}^{\circ} \mathrm{de} \\
\text { Presidencias }\end{array}$ & $\begin{array}{c}\% \text { de } \\
\text { presidencias }\end{array}$ & $\begin{array}{c}\text { Año primer y } \\
\text { última } \\
\text { presencia }\end{array}$ \\
\hline Balsebre Torroja, Armand & UAB & 13 & 7 & 7,14 & $1987-2007$ \\
\hline Barea Monge, Pedro & UPV & 9 & 2 & 2,04 & $1992-2005$ \\
\hline Cebrián Herreros, Mariano & UCM & 9 & 7 & 7,14 & $1985-20() 6$ \\
\hline Soengas Pérez, José & USC & 9 & 0 & 0 & $1995-2007$ \\
\hline Fernandez del Moral, A. Javier & UCM & 8 & 6 & 6,12 & $1995-2007$ \\
\hline Farias García, Pedro & UCM & 7 & 5 & 5,1 & 1994-2005 \\
\hline Merayo Pérez, Arturo & UCAM & 6 & 0 & 0 & $1991-2005$ \\
\hline Pedrero Esteban, Luis & UPS & 6 & 0 & 0 & 1999-2006 \\
\hline Prado Picó, Emiliox & UAB & 6 & 4 & 4,08 & $1984-2005$ \\
\hline Martí Martí, Josep María & UAB & 6 & 2 & 2,04 & $1988-2007$ \\
\hline \multicolumn{6}{|l|}{ - } \\
\hline Sanchez-Bravo, Antonio & UCM & 4 & 3 & 3,06 & $1983-2001$ \\
\hline
\end{tabular}

\subsection{Análisis de Redes Sociales}

La red del tribunal de oposiciones es una red muy amplia, con 339 actores enlazados por 1207 relaciones entre sí, de las cuales 1058 (87\%) tienen relaciones con valor 1 , y 149 (23\%) tienen relaciones con valores superiores. Los valores de densidad de la red son explicados en gran medida por la amplitud de esta. Es lógico que en una red pequeña haya mayor densidad y la vaya perdiendo conforme aquella va aumentando. La media de relaciones que tiene cada actor de la red es de 7.0791789 (el perfil normal es un actor que se relaciona con 7 personas diferentes, pero en relaciones débiles, ya que solamente el $23 \%$ de las relaciones tiene un valor superior a 1). Aparentemente que cada actor se relacione con 7 personas de media, puede ser un dato significativo, si no fuera porque en un solo tribunal participan 6 personas si sólo existe un director. Lo que indica que el número de relaciones base es de 5 (las tesis sin tribunales lógicamente no han sido incluidas en el Análisis de Redes).

Tabla 4. Académicos con mayor grado de interrelación. Periodo 1976-2008

\begin{tabular}{|c|c|}
\hline $\begin{array}{c}\text { NÚMERO DE } \\
\text { RELACIONES }\end{array}$ & ACTORES CON MAYOR INTERRELACIÓN \\
\hline 5 & $\begin{array}{r}\text { FrANQUET CALVET, ROSA } \\
\text { PRADO PICÓ, EMILIO }\end{array}$ \\
\hline 5 & $\begin{array}{c}\text { BALSEBRE TORROJA, ARMAND } \\
\text { PrADO PICÓ, EMILIO }\end{array}$ \\
\hline 5 & $\begin{array}{c}\text { FARIAS GARCÍA, PEDRO } \\
\text { VENTIN PEREIRA, JOSÉ AUGUSTO }\end{array}$ \\
\hline
\end{tabular}


El mayor valor de las relaciones es el que tienen las tres parejas de la tabla 4 que entre sí tienen relaciones de valor 5. Llama la atención que Emilio Prado Picó esté en dos de las relaciones con más densidad. Estos datos descriptivos generales de la red, nos hacen suponer que puede haber un grupo dentro de la red con relaciones más densas, formado principalmente por lo que en el análisis bibliométrico llamábamos "élite", el grupo con mayor productividad y presencia en tribunales.

\subsubsection{Medidades de centralidad: grado, cercanía e intermediación}

La tabla 5 indica los 5 actores de la red con mayor cercanía, entendida como la independencia para llegar a cualquier punto de la red sin apoyarse en otros actores. En la Tabla 5 se aprecia a los 5 actores con mayor grado de intermediación, mayor capacidad para conectar grupos y hacer de intermediarios, según Burt (2004) las élites en los análisis de intermediación se relacionan con la capacidad de innovación.

Tabla 5. Académicos con mayor grado de centralidad, medida en Grado, Cercanía e Intermediación

\begin{tabular}{|c|c|c|c|c|c|}
\hline \multicolumn{2}{|c|}{ Grado } & \multicolumn{3}{c|}{ Cercanía } & \multicolumn{2}{c|}{ Intermediación } \\
\hline $\mathbf{0 , 1 7 9}$ & Balsebre Torroja, Armand & $\mathbf{0 , 3 4 3}$ & Balsebre Torroja, A. & $\mathbf{0 , 1 3 3}$ & Balsebre Torroja, Armand \\
\hline $\mathbf{0 , 1 3 8}$ & Cebrián Herreros, Mariano & $\mathbf{0 , 3 3 7}$ & Barea Monge, Pedro & $\mathbf{0 , 1 0 1}$ & Merayo Pérez, Arturo \\
\hline $\mathbf{0 , 1 1 5}$ & Merayo Pérez, Arturo & $\mathbf{0 , 3 3 3}$ & Merayo Pérez, Arturo & $\mathbf{0 , 0 9 2}$ & Barea Monge, Pedro \\
\hline $\mathbf{0 , 1 0 9}$ & Prado Picó, Emilio & $\mathbf{0 , 3 3 3}$ & Soengas Pérez, José & $\mathbf{0 , 0 8 1}$ & Cebrián Herreros, Mariano \\
\hline $\mathbf{0 , 1 0 6}$ & Barea Monge, Pedro & $\mathbf{0 , 3 2 0}$ & Cebrián Herreros, M. & $\mathbf{0 , 0 5 6}$ & Prado Picó, Emilio \\
\hline
\end{tabular}

\subsubsection{Grafos}

Gráfico 4. Grafo Centralidad de Grado. Red de modo-1 de coparticipación en tribunales de tesis sobre Radio. Subgrupo Período 1978-2008

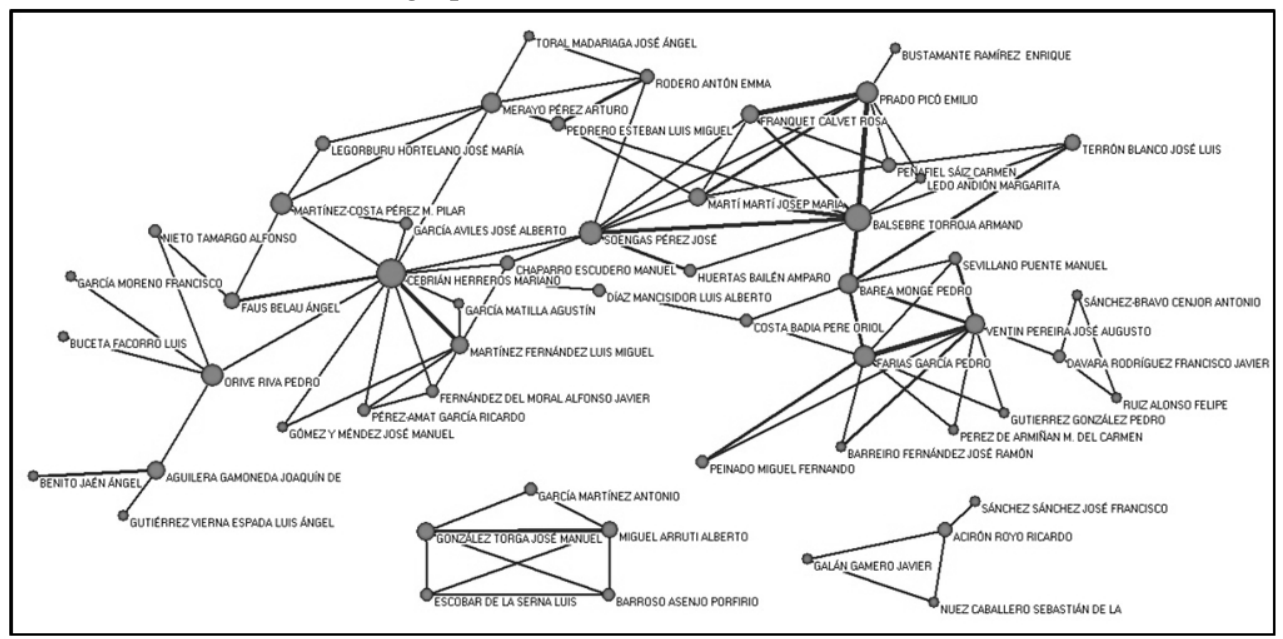

En la gráfica 4 ( red 1), la red está formada por 3 componentes. Los dos más pequeños están compuestos por 4 y 5 autores respectivamente. El componente de 4 actores tiene a Ricardo Acirón como el nodo con mayor valor central. Este grupo se forma a 
raíz de tesis doctorales relacionadas con la Radio como Empresa Informativa y la radio en Canarias. El factor de empresa informativa justifica la presencia de Javier Galán Gamero, y del fallecido Sebastián de la Nuez como experto en cultura y comunicación en Canarias. El componente de 5 miembros está formado principalmente por profesorado de la Universidad San Pablo CEU, a excepción de Porfirio Barroso y Antonio García Martínez, ambos profesores de la UCM. La tercera red, de 45 actores tiene como elementos centrales a Mariano Cebrián, Armand Balsebre, José Soengas, Pedro Barea y Pedro Orive. Es una red donde se ha representado el grado de los actores de la subred. El grado de la subred no representa el grado de la red, sino solamente las relaciones del grupo más cohesionado, aquellos que tienen relaciones con una frecuencia igual o superior a 2.

Gráfico 5. Grafo. Red de modo-2. Universidades con mayor producción y sus directores. Período: 1976-2008.

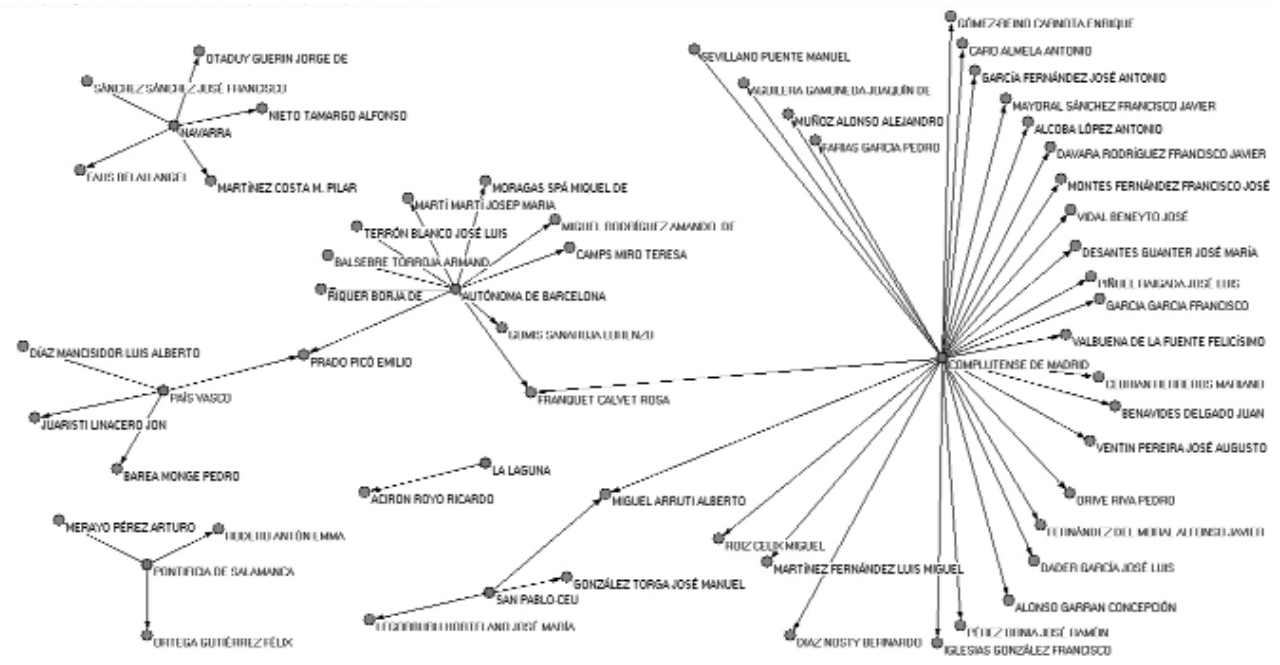

En último lugar, la gráfica 5, es una red de modo-2 que representa las 7 universidades con mayor productividad y sus directores. Se vuelve a poner de manifiesto la alta producción de la UCM y, más específicamente, el alto número de académicos que han dirigido tesis. La proporción de tesis/académicos de la Universidad Autónoma de Barcelona (UAB) es menor, lo que a rasgos generales puede indicar un grupo de académicos con mayor especialización en Radio en la UAB. La UCM aporta un total de 47 tesis sobre Radio dirigidas por 32 profesores diferentes, en cambio la UAB aporta 24 tesis dirigidas por 10 profesores. El ratio de tesis/profesor es un punto superior en la Universidad Autónoma de Barcelona ( $\mathrm{UCM}=1,47$ y $\mathrm{UAB}=2,4)$. Es importante resaltar la figura de Emilio Prado Picó, Rosa Franquet Calvet y Alberto Miguel Arruti como académicos que "unen" universidades, ya que han dirigido tesis para dos universidades diferentes. 


\section{Conclusiones}

La producción de tesis ha seguido un crecimiento constante hasta 2003, en el que las Universidades Históricas de Comunicación (Complutense, Autónoma de Barcelona, Navarra y Universidad del País Vasco en menor medida) han jugado un papel fundamental.

Es sin duda la Universidad Complutense la que lidera el ranking de producción de tesis, sin embargo la ratio tesis/profesor es mayor para profesores de la Universidad Autónoma de Barcelona. Los datos recuperados en TESEO indican que es José Augusto Ventín Pereira (UCM) el director que más tesis ha dirigido, seguido por Emilio Prado Picó (UAB). Sin embargo el ARS pone de manifiesto la figura de Armand Balsebre como el personaje con mayor poder central en grado, cercanía e intermediación.

Desde el punto de vista metodológico se aprecia la gran complementariedad del Análisis Bibliométrico y el Análisis de Redes Sociales. El primero se centra en los actores y sus atributos, mientras que el Análisis de Redes se centra en los actores y las relaciones. Se ha llegado a un mismo acuerdo sobre las figuras predominantes (poder) a través de las élites de la Presidencia en tribunales (Análisis Bibliométrico) y los actores con mayor centralidad (cercanía e intermediación).

\section{Referencias bibliográficas}

AGUDELO VÉLEZ, Diana María et al. (2002): Estado de la Investigación Psicológica en España: el grado de Doctor y la Investigación Académica (1976-1998): Una réplica. Infocop.

AGUILERA MOYANO, Miguel de (1998): "La investigación sobre comunicación en España: una visión panorámica". Comunicación \& Cultura, $\mathrm{n}^{\circ}$ 4, pp. 5-11.

ÁLVAREZ, Macu (1993): "Communication studies in Spain. An individual perspective", en FRENCH, David y RICHARDS, Michael (eds.): Media education across Europe. Londres: Routledge, pp. 46-63.

BARBOSA, Andrés (2000): "Diez años de investigación ornitológica en España a través de las tesis doctorales". Ardeola, vol. 47, n 2, pp. 273-278.

BURT, Ronald S. (2004). "Structurals holes and good ideas". American Journal of Sociology, vol. 10 (2), pp. 349-99.

BUSTAMANTE RAMÍREZ, Enrique (1980): El poder emisor en España. Madrid, Universidad Complutense de Madrid.

CENTENO, Carlos et al. (2001): "Tesis doctorales relacionadas con cuidados paliativos realizadas en la Universidad Española entre 1985 y 2000". Medicina paliativa, 8(4), pp. 181-189.

CIVERA MOLLA, Cristina y TORTOSA GIL, Francisco Manuel (2001): "Estado de la investigación psicológica en España: el grado de Doctor y la investigación académica (1976-1998)". Papeles del Psicólogo, 79, pp. 42-52.

DELGADO LÓPEZ-CÓZAR, Emilio (2002): La investigación en Biblioteconomía y Documentación. Gijon, TREA. 
DELGADO LÓPEZ-CÓZAR, Emilio (2003): "Las cifras de la Documentación en España". El profesional de la información. 12(5), pp. 334-367.

DELGADO LÓPEZ-CÓZAR, Emilio; TORRES SALINAS, Daniel; JIMÉNEZ CONTRERAS, Evaristo y RUIZ-PÉREZ, Rafael (2006): “Análisis bibliométrico y de redes sociales aplicado a las tesis bibliométricas defendidas en España (19762002): temas, escuelas científicas y redes académicas". Revista Española de Documentación Científica. 29 (4).

FERNÁNDEZ CANO, Antonio et al. (2003): "Análisis cienciométrico de las tesis doctorales españolas en Educación Matemática (1976-1998)”. Revista Española de Documentación Científica, 26 (2).

FERNÁNDEZ CANO, Antonio; TORRALBO RODRÍGUEZ, Manuel y VALLEJO RUIZ, Mónica (2008): "Revisión y prospectiva de la producción española en tesis doctorales de Pedagogía (1976-2006)". Revista de Investigación Educativa, 26(1), pp. 191-207.

FIGUEREDO, Eduardo et al. (2002): "Tesis doctorales españolas sobre Anestesiología y Reanimación”. Revista española de Anestesiología y Reanimación. 49(3), pp. 124-139.

FUENTES PUJOL, Eulalia y ARGUIMBAU VIVÓ, Llorenç (2010): "Las tesis doctorales en España (1997-2008): análisis, estadísticas y repositorios cooperativos". Revista Española de Documentación Científica. 33(1) pp. 63-89.

FUENTES PUJOL, Eulalia y GONZALEZ QUESADA, Alfonso (2002): “Tesis doctorales en España en Informacion y Documentacion". La documentación para la investigación. Homenaje a Jose Antonio Martin Fuertes. León. Universidad de León.

JONES, Daniel E. (1998): "Investigación sobre comunicación en España. Evolución y perspectivas". Zer. http://www.ehu.es/zer/zer5/1jones.html [fecha de consulta: 29 de enero de 2011]

JONES, Daniel E. (1999): "Investigaciones en España sobre la comunicación iberoamericana". Revista Comunicación y Sociedad, nº 35, pp. 229-268.

JONES, Daniel E. (2000): "Investigació sobre comunicació a Catalunya als anys noranta". Treballs de comunicació, (13-14), pp. 41-55.

JONES, Daniel E. (1994): "Investigació sobre comunicació al'Espanya dels noranta". En Cultura y comunicación social: América Latina y Europa ibérica. Barcelona, Centre d'Investigació de la Comunicació, pp. 87-98.

JONES, Daniel E. (1997): “Investigació sobre comunicació social a l'Espanya de les autonomies". Anàlisi. Quarderns de comunicació i cultura, 21, 101-120.

JONES, Daniel E. et al. (2000): "Investigación sobre comunicación en España. Aproximación bibliométrica a las tesis doctorales (1926-1998)". Barcelona, Com Cat, 152.

LÁZARO Y TORRES, María Luisa de (2002): "La Geografía a las puertas del tercer milenio a partir de las tesis doctorales leídas en los noventa". Anales de Geografia de la Universidad Complutense. pp. 49-65. 
LÓPEZ YEPES, José (2002): "Focos de investigación y escuelas científicas en Documentación a través de la realización y dirección de tesis doctorales. El caso del Departamento de Biblioteconomía y Documentación de la Universidad Complutense de Madrid (1983-2001)". Documentación de las Ciencias de la Información, (25), pp. 19-54.

MARTÍNEZ NICOLÁS, Manuel (2006): "Masa (en situación) crítica. La investigación sobre el periodismo en España: comunidad científica e intereses de conocimiento". Anàlisi, 33, pp. 135-170.

MUÑOZ TINOCO, Concepción y PEÑA ARREBOLA, Andrés (1998): “Tesis doctorales sobre rehabilitación presentadas en España en el período 1976-1996". Rehabilitación, 32(4), pp. 221-38.

PÉREZ DELGADO, Esteban y MESTRE, María Vicenta (1991): “La psicología en España a través de las tesis doctorales sobre psicología en las universidades españolas". Revista de Historia de la Psicología, 12(2), pp. 59.

PONCE DE LEÓN ELIZONDO, Ana María; GARBALLO IBORT, Esther y LOZA OLAVE, Edmundo (1998): "Análisis de las tendencias en las tesis doctorales de educación física. Cursos 1980-1981 a 1995-1996”. Educación Física y Deportes, 52, pp. 104-8.

TORRES RAMÍREZ, Isabel y TORRES SALINAS, Daniel, (2005): “Tesis doctorales sobre estudios de las mujeres en España (1976-2002): A propósito de un indicador definitivo en investigación". Revista Española de Documentación Científica, 28(4).

TORRES RAMÍREZ, Isabel y TORRES SALINAS, Daniel, (2007): Tesis doctorales sobre estudios de las mujeres en las universidades de España (1976-2005): Análisis bibliométrico y repertorio bibliográfico. Sevilla, Instituto Andaluz de la Mujer.

VALLEJO-RUIZ, Mónica; FERNÁNDEZ-CANO, Antonio; TORRALBO, Manuel; MAZ, Alexander y RICO, Luis (2008): "History of Spanish mathematics education focusing on $\mathrm{PhD}$ theses". International Journal of Science and Mathematics Education, 6(2), pp. 313-327. 